\title{
THE FUNCTIONS OPERATING ON CERTAIN ALGEBRAS OF MULTIPLIERS ${ }^{1}$
}

\author{
BY MISHA ZAFRAN
}

Communicated by A. P. Calderón, April 8, 1976

In this note, we announce a new result concerning functions operating on multiplier algebras. We begin by introducing the following notation. Let $G$ be a LCA group with dual group $\Gamma . M(G)$ will denote the algebra of finite, regular Borel measures on $G$. Let $M_{0}(G)=\{\mu \in M(G) \mid \hat{\mu}$ vanishes at $\infty$ on $\Gamma\}$. If $1 \leqslant$ $p<\infty$, let $M_{p}(G)$ denote the class of multiplier transformations on $L_{p}(G)$. If $T \in M_{p}(G), \hat{T}$ will be the unique function in $L_{\infty}(\Gamma)$ so that $T(f)^{\wedge}=\hat{T} \hat{f}$, for all integrable simple functions $f$. Finally, we write $C_{0} M_{p}(G)=\left\{T \in M_{p}(G) \mid \hat{T}\right.$ is continuous and vanishes at $\infty$ on $\Gamma$ \}.

Suppose that $G$ is nondiscrete. It is well known that only entire functions operate on the Banach algebra $M(G)[3$, Chapter 6]. This result was strengthened in [1]. There, Igari showed that only entire functions operate from $M(G)$ into the algebra $M_{p}(G), 1<p<\infty, p \neq 2$. In [4], Varopoulos showed that for compact $G$, only entire functions operate on $M_{0}(G)$. We have the following theorems, which, in a sense, may be viewed as the $L_{p}$ analogues of the aforementioned result of Varopoulos.

Theorem 1. Let $1<p<\infty$ with $p \neq 2$. Suppose that $F:[-1,1] \rightarrow \mathbf{C}$ and that $F$ operates on the algebra $C_{0} M_{p}\left(\mathrm{~T}^{n}\right)$. Then $F$ coincides with an entire function in some neighborhood of 0 .

THEOREM 2. Let $1<p<\infty$ with $p \neq 2$, and let $G$ denote one of the groups $\mathbf{R}^{n}$ or $\mathbf{Z}^{n}$. Suppose that $F:[-1,1] \rightarrow \mathbf{C}$ and that $F$ operates on the algebra $C_{0} M_{p}(G)$. Then $F$ coincides with an entire function on $[-1,1]$.

These results complete the investigation begun by the author in [5]. We now indicate some of the ideas involved in the proof.

Assume that $G=\mathbf{T}$ and $1<\mathrm{p}<2$. By standard arguments (see [1] and [3, Chapter 6]) we may assume that $F(x)=\Sigma_{k=1}^{\infty} a_{k} x^{k}$ for $|x|<\epsilon$. It then suffices to show that there exists $j_{\epsilon}$ such that

$$
\left|a_{j}\right| \leqslant C_{\epsilon} 10^{j}
$$

for all $j \geqslant j_{\epsilon}$. This is accomplished by studying refinements of the multipliers considered in [5]. Corresponding to the sequence $\left\{a_{j}\right\}$, we construct measures $\left\{\lambda_{j}\right\}, \lambda$ in $M(\mathrm{~T})$ so that for all $j$

AMS (MOS) subject classifications (1970). Primary 42A18.

1 This work was supported in part by NSF Grant GP $28112 \mathrm{~A}$. 


$$
\hat{\lambda}_{j} \text { is real-valued and }\left\|\hat{\lambda}_{j}\right\|_{\infty} \leqslant 2^{1 / 2} 2^{-n(j) / 2} \text {, }
$$

$$
\left|\lambda_{j}\right| \leqslant \lambda
$$$$
\left\|\sum_{k=1}^{j} a_{k} 2^{n(j) k / p^{\prime}} \lambda_{j}^{k}\right\|_{M_{p}} \geqslant c\left(j ! / j^{j}\right) 2^{-j / p^{\prime}}\left|a_{j}\right| .
$$

Here $\{n(j)\}$ is a sequence of positive integers tending to infinity and $1 / p+$ $1 / p^{\prime}=1$. As in [5], the measures $\left\{\lambda_{j}\right\}$ and $\lambda$ are essentially obtained as "generalized Riesz products" of combinations of certain Rudin-Shapiro measures. Inequalities (4) is proved by carefully studying the combinatorial properties of these Rudin-Shapiro measures. Moreover, the special properties of Rudin-Shapiro polynomials makes it possible, in essence, to "ignore" the term $\Sigma_{k=j+1}^{\infty} a_{k} 2^{n(j) k / p^{\prime}} \lambda_{j}^{k}$ when estimating $\left|a_{j}\right|$. We now define $U\left\{f_{j}\right\}=\left\{2^{n(j) / p^{\prime}} \lambda_{j} * f_{j}\right\}$. Then $U$ is a bounded operator on $L_{p}\left(l_{2}\right)$. We construct our basic multiplier $T$ by "cutting off" and "piecing together" the measures $2^{n(j) / p^{\prime}} \lambda_{j}$ via the Littlewood-Paley theory (see [5] for details). The estimate (1) then follows by studying the multiplier $F(T)$, and using the properties of $\left\{\lambda_{j}\right\}$. This will prove Theorem 1 for the circle groups.

Theorems 1 and 2 now follow by rather standard arguments for the cases $G=\mathbf{T}^{n}$ or $G=\mathbf{R}^{n}$. However, the case $G=\mathbf{Z}^{n}$ (more particularly, if $G=\mathbf{Z}$ ) is more difficult and requires some additional ideas.

We prove the theorem for the integer group by constructing a multiplier $S \in C_{0} M_{p}(\mathbf{R})$ so that $\hat{S}$ is real-valued, supp $\hat{S} \subseteq[0,1]$, and so that the behavior of $\hat{\mathrm{S}}$ near the origin reflects the behavior of our basic multiplier $\hat{T}$ near $\infty$. The construction consists in combining the method outlined above, with a technique of Igari [2].

The proof is essentially in the same spirit as that indicated for $G=\mathbf{T}$. However, the arguments are much more involved. In particular, we introduce a vector-valued analogue of the space BMO. This allows us to obtain sharp $L_{p}$ estimates for the operators involved in our constructions.

Detailed proofs of the ideas sketched here will appear in [6].

\section{REFERENCES}

1. S. Igari, Functions of $L^{p}$ multipliers, Tôhoku Math. J. 21 (1969), 304-320.

2. - Functions of $L^{p}$ multipliers. II, Tôhoku Math. J. 26 (1974), 555-561.

3. W. Rudin, Fourier analysis on groups, Interscience, New York, 1967. MR 27 \#2808.

4. $\mathrm{N}$. Varopoulos, The functions that operate on $B_{0}(\Gamma)$ of a discrete group $\Gamma$, Bull. Soc. Math. France 93 (1965), 301-321. MR 24 \#565.

5. M. Zafran, The spectra of multiplier transformations on the $L_{p}$ spaces, Ann. of Math. (2) 103 (1976), 355-374.

6. - The functions operating on multiplier algebras, J. Functional Analysis (to appear) 